\title{
Bilateral Spontaneous Urinoma in a Patient with Primary Hypothyroidism
}

\author{
Mohd. Ismail ${ }^{1}$, Syed Arshad Mustafa ${ }^{2}$, Afroza Jan ${ }^{3}$, Saquib Zaffar ${ }^{4}$ \\ Received: November 16, 2015 Accepted: August 31, 2016 \\ doi: http://dx.doi.org/10.3329/jemc.v6i3.29686
}

\begin{abstract}
Urinomas are a rare entity involving collection of urine in the perinephric and adjacent spaces resulting from disruption of the urinary collecting system at any level from the calyx to the urethra. Among other causes, blunt trauma to the abdomen is one of the commonest causes. Literature regarding medical causes leading to urinomas is scarce. We report a case of a patient with spontaneous bilateral urinoma with co-incident hypothyroidism.
\end{abstract}

Key words: Flank pain; Urinoma; Hypothyroidism

J Enam Med Col 2016; 6(3): 166-168

\section{Introduction $^{1-4}$}

Urogenital trauma is the most common cause of urinary leaks from the kidney, ureter, bladder and urethra. ${ }^{1}$ The formation of urinomas is insidious initially and may lead to complications such as abscess formation and electrolyte imbalances if not promptly diagnosed and appropriately managed. $^{2}$ Urinomas have been reported in literature in association with pelvic masses, urinary calculus, posterior urethral valves, ureteropelvic junction (UPJ) obstruction, congenital anomalies, chronically distended bladder resulting in obstructive uropathy. ${ }^{3}$ Though data of spontaneous urinomas in association with medical diseases like hypothyroidism are scarce, a rare case of hypothyroid ascites responding to thyroxine replacement has been reported. ${ }^{4}$ Possible pathophysiology of spontaneous urinoma is raised intrapelvicalyceal pressure resulting in back-flow of urine with subsequent multiple rupture or porosities at calyceal fornices and extravasation of urine through sinus and renal capsule. ${ }^{3}$

\section{Case report}

A 40-year-old female presented to outpatient department with two weeks history of pain in both flanks. It was dull aching and continuous in nature with no constitutional symptoms like fever, dysuria or increased frequency of micturition. There was no history of antecedent trauma, diabetes, tuberculosis, hypertension or any significant surgical or medical past history. Physical examination revealed normal vitals. There was mild pallor but no cyanosis, jaundice or thyromegaly. There was no abdominal tenderness, organomegaly or ascites.

Baseline investigation revealed hypochromic microcytic anemia with haemoglobin of $9.1 \mathrm{gm} \%$, mean corpuscular volume (MCV) 72, mean corpuscular haemoglobin concentration (MCHC) of 25 and mean corpuscular haemoglobin (MCH) 24. Renal function tests and liver function tests were normal. Urine routine examination was normal and urine culture was sterile. Electrocardiography and chest radiography were normal. Transabdominal ultrasonography revealed bilateral perinephric collection with a maintained corticomedullary differentiation, normal hepatobiliary system and no ascites.

1. Assistant Professor, Department of Internal Medicine, Government Medical College \& Associated Hospitals, Srinagar

2. Lecturer, Department of Radiation Oncology, Government Medical College \& Associated Hospitals, Srinagar

3. Registrar, Department of Internal Medicine, Government Medical College \& Associated Hospitals, Srinagar

4. Registrar, Department of Radiation Oncology, Government Medical College \& Associated Hospitals, Srinagar

Correspondence Syed Arshad Mustafa, E mail: syedarshad07@gmail.com 


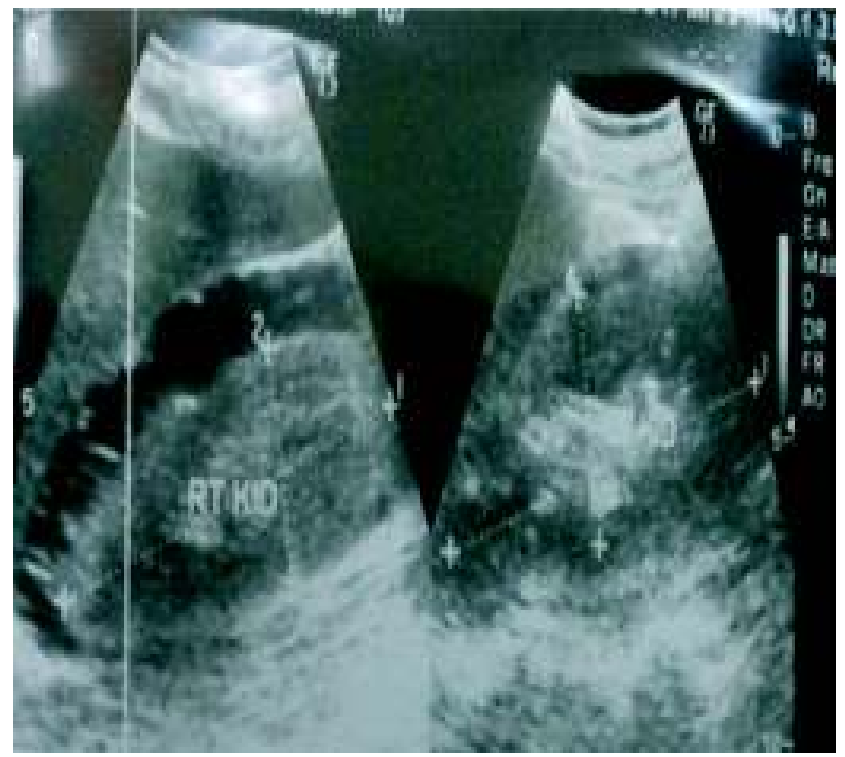

Fig 1. USG of abdomen showing bilateral perinephric collection

Thyroid profile showed raised thyroid stimulating hormone (TSH) of 39.6 [NR 3.5-5.5], decreased free T4, serum calcium $8.9 \mathrm{mg} \%$, phosphorus $4.5 \mathrm{mg} \%$. Urine smear for acid fast bacillus was negative. Contrast enhanced computed tomography abdomen and pelvis showed bilateral perinephric collection. There was no hydronephrosis, hydroureter, ascites, organomegaly or retroperitoneal mass. Magnetic resonance urography revealed no evidence of ureteric obstruction in the pelvicalyceal system; bilateral perinephric collection was seen.

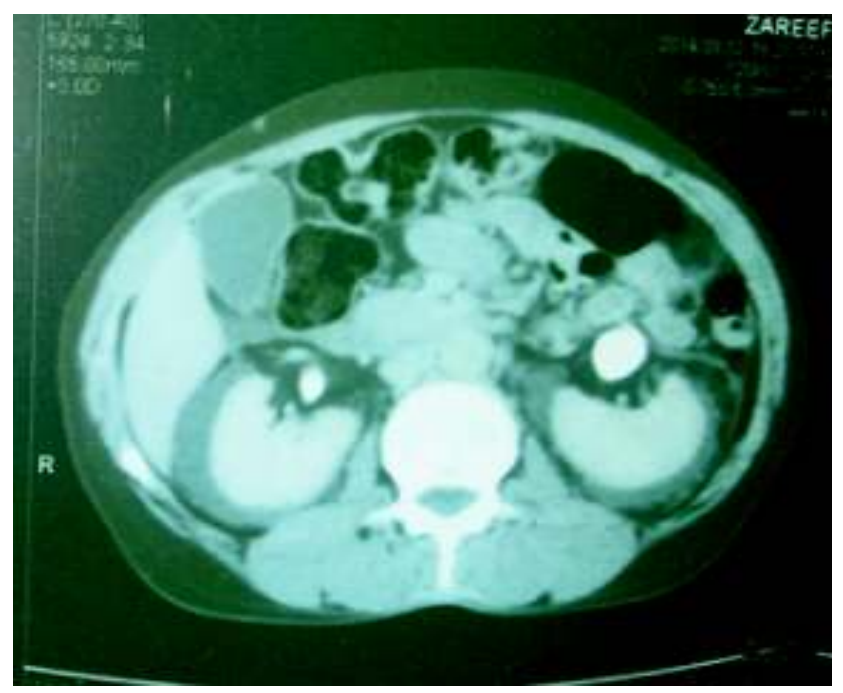

Fig 2. Contrast enhanced CT of abdomen and pelvis showing bilateral perinephric collection

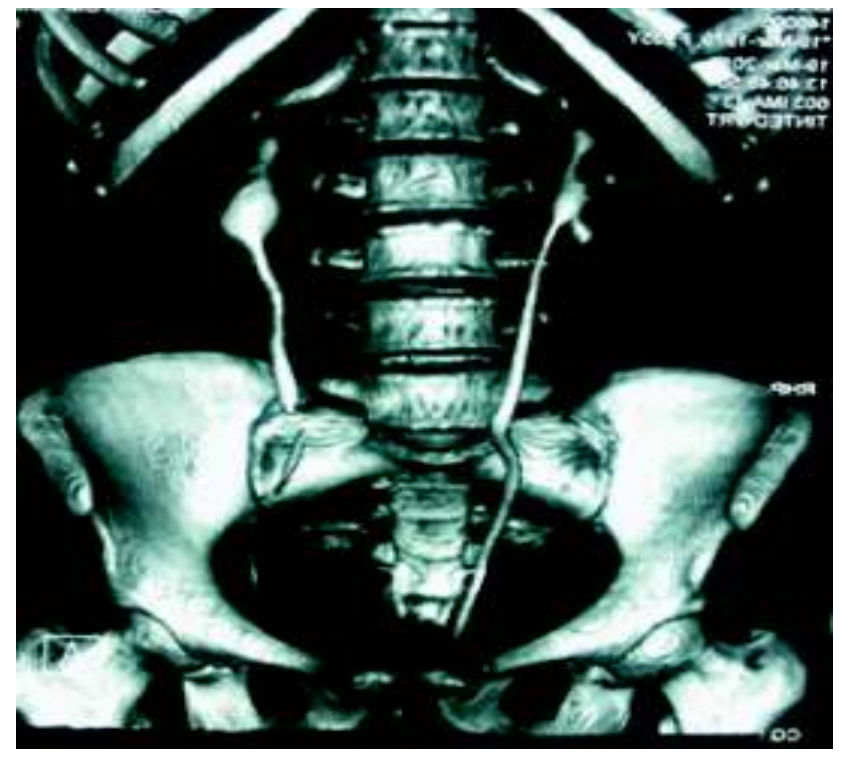

Fig 3. Magnetic resonance urography showing bilateral perinephric collection

USG-guided aspiration of perinephric fluid revealed straw coloured fluid with no M-cells, lymphocytes, proteins or sugar. Creatinine concentration of the fluid was $9.08 \mathrm{mg} \%$ confirming it to be urine. Patient was managed conservatively with monthly follow-up during which transabdominal sonography was done each time for consecutive three months. Patient was incidentally found to be hypothyroid on routine investigations for which he was put on thyroxine $75 \mu \mathrm{g} /$ day. At the end of three months there was complete resolution of the collection.

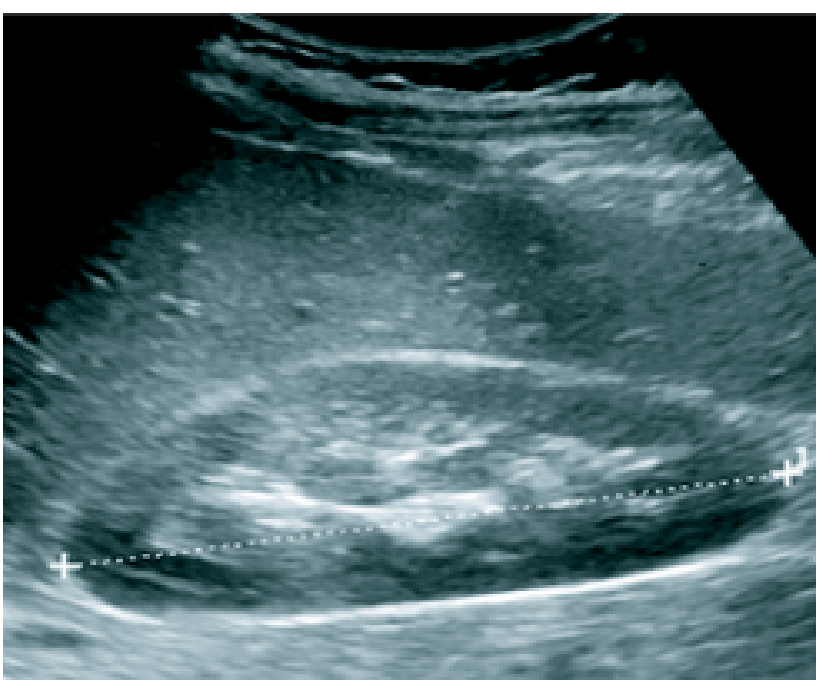

Fig 4. USG of abdomen showing complete resolution of collection 
Whether the resolution was affected by thyroxine replacement for hypothyroidism warrants further study, as there was no other medical or surgical comorbidity that may be correlated with presentation of urinoma in this patient.

\section{Discussion}

A urinoma, also called pararenal pseudocyst, is an encapsulated collection of extravasated fluid and typically found in the area adjacent to the kidneys ${ }^{5,6}$ or extends into the retroperitoneum ${ }^{7}$. A urinoma is the result of a breach of the integrity of the pelvis or calices of the kidney or of the ureter. ${ }^{8}$ The urine collection in the perirenal fat causes an inflammatory response with lipolysis resulting in its fibrous encapsulation. Urinomas are usually caused by blunt trauma to the kidneys. While extravasation of urine is common as a result a severe blunt trauma (2-18\%), spontaneous resolution is typical and urinoma formation develops only in few instances. ${ }^{9}$ Less common causes of urinoma development are ureteral obstructions due to cancer, calculus formation, pregnancy, congenital causes or idiopathic retroperitoneal fibrosis. ${ }^{5-7}$ Urinomas tend to develop gradually. Symptoms depend on size and location of the lesion. ${ }^{7}$ Eventually local pain and pressure symptoms may become apparent. Biochemical testing of renal function is indicated. Imaging (IVP, ultrasonography, CT scan) will identify the lesion. Image-guided percutaneous needle aspiration is both diagnostic and therapeutic. ${ }^{6}$ If left untreated, complications may arise including abscess formation, peritonitis, sepsis and damage to the urinary tract by fibrosis and granuloma formation. It is recommended, as a first step, to drain the lesion with ultrasound or CT guidance. $^{5,6}$ If a patient has an underlying obstructive problem, it needs to be addressed according to its aetiology.

\section{Acknowledgement}

We are highly thankful to the department of radiology for taking exceptional interest in pursuing this rare case.

\section{References}

1. Titton RL, Gervais DA, Hahn PF, Mukesh G. Harisinghani MG, Arellano RS et al. Urine leaks and urinomas: diagnosis and imaging-guided intervention. Radiographics 2003; 23(5): 1133-1147.

2. Lukacz ES, Nager CW. Ureteral injury presenting with hyponatremia. Obstet Gynecol 2001; 98: 974-976 .

3. Kumar A, Suman SK, Khan I, Pridarshi S. Spontaneous perinephric urinoma in a postpartum woman: case report and review. Int J Sci Stud 2014; 2(6): 154-156.

4. Philips CA, Sinha U, Chattopadhyay P, Mukhopadhyay P, Haldar S. Isolated ascites in hypothyroidism: medical and ethical issues. Journal of Indian Med Assoc 2010; 108(8): 523-524.

5. Ketabchi AA, Ketabchi M, Narkam M. Percutaneous drainage of a late-onset giant posttraumatic urinoma. Urol J 2009; 6: 214-216.

6. Rizvi S, Ibne A, Siddiqui MA, Syed M. A case report: urinoma as initial presenting sign of bladder malignancy. Indian Journal of Cancer 2011; 48: 514-517.

7. Ona-Olapo A, Oke T, Surenthiran S. Case reports: perirenal urinoma formation in a patient with a complex pelvic cyst. BJU Int. 2010. Available at: http://www.bjui.org/ ContentFullItem.aspx?id=547. Accessed May 2015.

8. Morano JL, Burkhalter JL. Percutaneous catheter drainage of post-traumatic urinoma. J Urol 1985; 134: 319-321.

9. Srinath N, Sood R, Rana KVS, Madhusoodhanan P. Urinoma following blunt renal trauma. MJAFI 2000; 56: 3446 . 